\title{
Inhibition of reactive oxygen species generation attenuates TLR4-mediated proinflammatory and proliferative phenotype of vascular smooth muscle cells
}

\author{
Yan $\mathrm{Pi}^{1}$, Li-li Zhang ${ }^{1}$, Bing-hu Li, Lu Guo, Xiao-jie Cao, Chang-yue Gao and Jing-cheng Li
}

\begin{abstract}
Reactive oxygen species (ROS) are associated with inflammation and vasculature dysfunction. This study aimed to investigate the potential role of the ROS on vascular Toll-like receptor 4 (TLR4)-mediated proinflammatory and proliferative phenotype of vascular smooth muscle cells (VSMCs). A wire-induced carotid injury model was used in male TLR4-deficient (TLR4 ${ }^{-1-}$ ) and wild-type C57BL/6J mice to induce neointima formation. In the presence or absence of the ROS scavenger apocynin for 14 days, increased TLR4 and proinflammatory cytokines were observed in wire injury-induced carotid neointima and in platelet-derived growth factor-BB (PDGF-BB)-stimulated VSMCs. The TLR4 ${ }^{-1-}$ protected the injured carotid from neointimal formation and impaired the cellular proliferation and migration in response to PDGF-BB. Apocynin attenuated intimal hyperplasia. Pre-treatment with apocynin significantly inhibited intracellular ROS generation, accompanied by a significant suppression of TLR4 and proinflammatory cytokines expression, and VSMC proliferation and migration. However, the results were not obvious in TLR4 ${ }^{-/-}$condition. These findings highlight the importance of ROS inhibition in TLR4-mediated proinflammatory and proliferative phenotype of VSMCs, and suggest ROS as an essential therapeutic target for TLR4-associated vascular inflammation and vascular diseases.

Laboratory Investigation (2013) 93, 880-887; doi:10.1038/labinvest.2013.79; published online 17 June 2013
\end{abstract}

KEYWORDS: inflammation; intimal hyperplasia; reactive oxygen species; Toll-like receptor 4; vascular smooth muscle cell

Vascular smooth muscle cell (VSMC) proliferation and migration has been accepted as a common event in the pathophysiology of many vascular diseases, including atherosclerosis, atherogenesis and intimal hyperplasia. ${ }^{1}$ The quiescent VSMCs in adult vessels can be activated and regain the capability of proliferation and migration in response to injury, inflammation and stretch, and ultimately contribute to the formation of neointima. ${ }^{2}$ Suppression of VSMC proliferation and migration represents an important target point for therapeutic intervention to attenuate vascular diseases and prevent restenosis after vascular reconstructive procedures.

Reactive oxygen species (ROS) are involved in many pathophysiological processes of VSMCs, such as growth, proliferation, migration and differentiation. ${ }^{3-5}$ The main sources of ROS are NADPH oxidase, xanthine oxidase, lipoxygenase, mitochondria and the uncoupling nitric oxide synthase. ${ }^{6}$ ROS derived from VSMCs are involved in growth signaling pathways and have an important role in the pathogenesis of several vascular diseases associated with the proliferation and migration of VSMCs, such as atherosclerosis and neointima growth after angioplasty or vascular stenting. ${ }^{7-10}$ Studies have shown that intimal VSMCs in hyperplastic and atherosclerotic lesions exhibit high levels of ROS. ${ }^{11,12}$ However, despite intensive research efforts, the precise mechanisms underlying the effect of ROS remain to be fully elucidated.

An inflammatory response is widely accepted as an essential event in VSMC activation and intimal hyperplasia that occurs early in atherosclerosis. ${ }^{13,14}$ Many studies have shown that ROS overproduction is associated with intimal hyperplasia and atherosclerosis. ${ }^{15}$ In addition, ROS participate in initiating acute inflammatory responses and ultimately lead to vasculature dysfunction. Inhibition of the inflammatory response by ROS scavenger has been increasingly examined in recent studies. ${ }^{13}$ Toll-like receptor 4 (TLR4) is well known to have a critical role in initiating inflammation through increasing the production of proinflammatory factors, including interleukin (IL)-1, IL-6, interferon $\gamma$ and tumor

Department of Neurology, Institute of Surgery Research, Daping Hospital, Third Military Medical University, Chongqing, China

Correspondence: Professor J-c Li, MD, Department of Neurology, Institute of Surgery Research, Daping Hospital, Third Military Medical University, 10 Changjiang Branch Road, Yuzhong District, Chongqing 400042, China.

E-mail: lijingcheng11@hotmail.com

${ }^{1}$ These authors contributed equally to this work.

Received 13 March 2013; revised 27 April 2013; accepted 20 May 2013 
necrosis factor $\alpha$ (TNF- $\alpha),{ }^{16,17}$ and may make a fundamentally significant contribution to the crucial pathophysiological relationship between inflammation and vascular disorders. ${ }^{18}$ TLR4 is also reported to be involved in the modulation of VSMC proliferation, migration and neointimal formation. ${ }^{17,19}$ Studies have shown that TLR4 is expressed in the endothelial cells and upregulated by ROS. ${ }^{20,21}$ However, it remains unknown whether inhibition of ROS could ameliorate TLR4-mediated inflammation, VSMC proliferation and migration, intimal hyperplasia, and atherosclerosis.

In this study, we have investigated the essential role of ROS on vascular TLR4 expression and neointimal formation. In addition, we have tested the hypothesis that ROS promote VSMC proliferation and migration by enhancing TLR4-mediated inflammation, and eventually lead to intimal hyperplasia after wire injury, and inhibition of ROS generation could restrain this progress.

\section{MATERIALS AND METHODS Reagents}

Dulbecco's modified Eagle's medium (DMEM) and fetal bovine serum (FBS) were purchased from Gibco BRL (Carlsbad, CA, USA). Apocynin, recombinant murine platelet-derived growth factor-BB (PDGF-BB), methyl thiazolyl diphenyl-tetrazolium (MTT) and dimethyl sulfoxide were obtained from Sigma-Aldrich (St Louis, MO, USA). Antibodies targeting TLR4, GAPDH and smooth muscle $\alpha$-actin $(\alpha$-SMA) were from Santa Cruz Biotechnology (Santa Cruz, CA, USA). 2', $7^{\prime}$-Dichlorofluorescin diacetate (DCFH-DA) was purchased from Molecular Probes (Eugene, OR, USA).

\section{Animals}

Male TLR4-deficient mice (C57BL/6J background) and wildtype mice (WT, C57BL/6J) were obtained at 8-10 weeks of age from the Jackson Laboratory (Bar Harbor, ME, USA). A wire-induced carotid injury model was used in this study to induce intimal hyperplasia as described previously. ${ }^{22}$ Mice were briefly anesthetized with an intraperitoneal injection of $60 \mu \mathrm{g} / \mathrm{g}$ body weight sodium pentobarbital, and repeated intraperitoneal injections ( $12 \mu \mathrm{g} / \mathrm{g}$ body weight) were given as needed to maintain anesthesia. Mice displayed full sedation, no response to pain stimuli, muscle relaxation, even breathing and pink oral mucosae, and were well anesthetized and ready for surgery.

To determine the effects of ROS scavenger on intimal hyperplasia, apocynin ${ }^{23}$ was administered orally $(200 \mathrm{mg} / \mathrm{kg} /$ day by gavage) for 14 days starting from the day of wire injury. All the animals had ad libitum access to food and water. Mice were killed at 14 days after wire injury, blood serum was collected and carotid injured arteries were perfusion fixed in situ with $4 \%$ formaldehyde solution $(\mathrm{pH}$ 7.4) and then harvested. The arterial segments were embedded in paraffin and cut at eight to ten sites $(600 \mu \mathrm{m}$ apart). Sections of $6 \mu \mathrm{m}$ thickness were prepared for morphometric and immunohistochemical analysis.

\section{Ethics Statement}

The experimental protocol used in the study conformed to the Guide for the Care and Use of Laboratory Animals published by the US National Institutes of Health and was approved by the Animal Research Committee of the Third Military Medical University.

\section{Histopathology Analysis}

Morphometric analysis was performed on hematoxylineosin-stained slides. The area of each vascular layer was measured by tracing the external elastic lamina, internal elastic lamina and vessel lumen. The intimal area was determined by subtracting the lumen area from the area within the internal elastic lamina. Media area was considered as the area between the external elastic lamina and internal elastic lamina. The intimal hyperplasia was assessed by calculating the ratio of intima to media area.

For immunohistochemistry, sections were deparaffinized and rehydrated by serial immersion in xylene, alcohol and water. Thereafter, $3 \%$ hydrogen peroxide was used to block endogenous peroxidase. Nonspecific binding was blocked with $0.5 \%$ blocking solution. $\alpha$-SMA was used as a protein marker for VSMCs and was applied at a dilution of 1:100 for $1 \mathrm{~h}$ at room temperature, followed by amplification with secondary antibodies, stained with DAB and counterstained with hematoxylin then visualized.

\section{Cell Culture}

VSMCs were isolated from the thoracic aorta of TLR4 ${ }^{-1-}$ and C57BL/6J mice using an explant technique and cultured in DMEM supplemented with $10 \%$ FBS, $100 \mathrm{U} / \mathrm{ml}$ penicillin and $100 \mathrm{U} / \mathrm{ml}$ streptomycin in air supplemented with $5 \%$ $\mathrm{CO}_{2}$ at $37^{\circ} \mathrm{C}$. Cultures were confirmed to consist primarily (>95\%) of VSMCs both morphologically by their classical 'hill and valley' appearance, and immunohistochemically by $\alpha$-SMA immunoreactivity. Cells in the second to sixth passages were used for experiments.

ROS generation was pharmacologically manipulated respectively by PDGF-BB and apocynin. Before exposure to $20 \mathrm{ng} / \mathrm{ml}$ PDGF-BB, VSMCs were pre-treated for $30 \mathrm{~min}$ with apocynin $(100 \mu \mathrm{mol} / \mathrm{l}){ }^{24-26}$ Untreated cells were served as controls. Cells and their supernatants and lysates were collected for ROS detection, enzyme-linked immunosorbent assay (ELISA) and western blot, respectively.

\section{Measurement of ROS}

Intracellular ROS were determined by oxidative conversion of cell permeable DCFH-DA to fluorescent dichlorofluorescein (DCF) as previously described. ${ }^{27}$ Briefly, VSMCs with $70 \%$ confluency were washed with serum-free DMEM and incubated in the dark with DCFH-DA $10 \mu \mathrm{mol} / \mathrm{l}$ for $20 \mathrm{~min}$ at $37^{\circ} \mathrm{C}$. Cells were then trypsinized, resuspended in PBS and subjected to flow cytometry analysis (Calibur, Becton Dickinson). The mean of DCF fluorescence intensity was obtained from 10000 events. Cells were excited at $488 \mathrm{~nm}$, 
and DCF fluorescence was read on FL1 $(530 \pm 15)$ in $\log$ scale.

\section{ELISA}

IL- $1 \beta$, IL- 6 and TNF- $\alpha$ were measured both in the serum of mice and in the supernatants of VSMCs by ELISAs according to the manufacturer's instructions (R\&D Systems, Minneapolis, MN, USA).

\section{Western Blot Analysis}

Western blot analysis was used to determine the levels of TLR4. Protein samples were obtained either from homogenized arteries or cultured cells, and the protein concentration was determined. Protein samples $(30 \mu \mathrm{g})$ were subjected to SDS-PAGE, transferred to a nitrocellulose membrane. The membranes were probed with rabbit antiTLR4 antibody at 1:1000, and then incubated with horseradish peroxidase-conjugated anti-rabbit IgG. The proteins were detected by enhanced chemiluminescence (NEN, MA, USA) and quantified using a Gel Doc 2000 Imager (Bio-Rad, CA, USA). Western blot quantification was performed by densitometry and normalized to GAPDH.

\section{MTT Assay}

Cell proliferation was determined by MTT assay. Briefly, VSMCs were plated on a 96-well plate at $3 \times 10^{4}$ cells per well, cells were incubated with PDGF-BB $(20 \mathrm{ng} / \mathrm{ml})$ for $24 \mathrm{~h}$ in the presence or absence of pre-treatment with apocynin $(100 \mu \mathrm{mol} / \mathrm{l})$ for $1 \mathrm{~h}$. After incubation, a $20 \mu \mathrm{l}$ aliquot of $5 \mathrm{mg} / \mathrm{ml} \mathrm{MTT}$ dye was added, and the cells were incubated for $4 \mathrm{~h}$ at $37^{\circ} \mathrm{C}$. After the 4 -h incubation, $150 \mu \mathrm{l}$ of DMSO was added to the cells and mixed thoroughly. The cells were agitated on a plate shaker for $5 \mathrm{~min}$ and absorbances were measured with a microplate reader (Bio-Rad) at $570 \mathrm{~nm}$.

\section{BrdU Assay}

DNA synthesis was measured by BrdU Cell Proliferation ELISA (Roche Diagnostic, Mannheim, Germany). VSMCs were plated on a 96-well plate at $2 \times 10^{3}$ cells per well, cells were incubated with PDGF-BB $(20 \mathrm{ng} / \mathrm{ml})$ for $24 \mathrm{~h}$ in the presence or absence of pre-treatment with apocynin $(100 \mu \mathrm{mol} / \mathrm{l})$ for $1 \mathrm{~h}$. BrdU (10 mM) was added and incubated for $8 \mathrm{~h}$. At the end of the incubation period, the medium was removed, cells were fixed, denaturated and probed with anti-BrdU antibody as suggested by the manufacturer's manual. The optical densities were measured at $490 \mathrm{~nm}$.

\section{Cell Migration Assay}

Cell migration was examined using modified Boyden chambers as described previously. ${ }^{17}$ Briefly, VSMCs $\left(5.0 \times 10^{4}\right.$ cells $\left./ \mathrm{ml}\right)$ were plated in the upper chamber with or without pharmacological intervention (apocynin, $100 \mu \mathrm{mol} / \mathrm{l}$ ). DMEM containing $0.4 \%$ FBS and PDGF-BB $(20 \mathrm{ng} / \mathrm{ml})$ was added to the lower chamber. The cells were then allowed to migrate for $24 \mathrm{~h}$ at $37^{\circ} \mathrm{C}$. Thereafter, cells on the underside of the filters were fixed with methanol and stained with hematoxylin, and then counted from five high-power $(\times 400)$ fields per well. The average was used as the migratory cell number.

\section{Statistical Analysis}

Statistical analysis was performed using GraphPad Prism 5 software (GraphPad Software Inc., La Jolla, CA, USA) or SPSS 13.0 (SPSS Inc., Chicago, IL, USA). Data are expressed as mean \pm s.e.m. of at least three independent experiments. Multiple-group statistical analyses were performed by one-way ANOVA followed by least significant difference post hoc testing. Differences were considered statistically significant at $P<0.05$.

\section{RESULTS \\ Intimal Hyperplasia in Wire-Injured Arteries}

Wire was used to induce carotid injury in male TLR4 ${ }^{-1-}$ and WT mice. After 14 days, the intima/media ratio was increased in injured carotid arteries compared with arteries from sham-operated WT mice. In contrast, TLR $4^{-1-}$ mice displayed very low intima/media ratio in response to carotid injury. Apocynin treatment effectively suppressed neointimal formation induced by wire injury in WT mice, but exerted no obvious effect on that in TLR4 ${ }^{-1-}$ mice (Figure 1a).

In agreement with our previous study, ${ }^{17}$ VSMCs, identified by $\alpha$-SMA-positive immunostaining, were found to make a major contribution to the formation of neointima (Figure 1b).

\section{Intracellular ROS Generation in VSMCs}

We examined the VSMC ROS generation in response to PDGF-BB. Intracellular release of ROS from VSMCs was measured in cells treated for $60 \mathrm{~min}$ with a single dose of PDGF-BB (20 ng/ml), which is known to generate intracellular ROS. ${ }^{24}$ ROS concentration was measured by flow cytometry using DCFH-DA. ${ }^{27}$ Compared with untreated VSMCs, treatment with PDGF-BB caused a significant increase in intracellular ROS generation. Pre-treatment of VSMCs with apocynin $(100 \mu \mathrm{mol} / \mathrm{l})$ for $30 \mathrm{~min}$ strikingly suppressed PDGF-BB-induced ROS generation (Figure 2).

\section{TLR4 Expression is Dependent on the Generation of ROS}

To determine the associations between ROS generation and TLR4 expression, we examined the effects of ROS on TLR4 expression. It was shown that wire injury increased the expression of TLR4 in vascular tissues taken from WT mice. Moreover, apocynin treatment significantly impeded the upregulation of TLR4 induced by wire injury (Figure 3a).

We also detected the potential impact of ROS on TLR4 expression in WT VSMCs. As shown in Figure 3b, exposure of VSMCs to PDGF-BB showed a significant increase in TLR4 expression in WT VSMCs, and this could be effectively inhibited by apocynin. These data suggest an important role of ROS in TLR4 expression, the upregulation of TLR4 in response to vascular injury or PDGF challenge is dependent on ROS generation. 

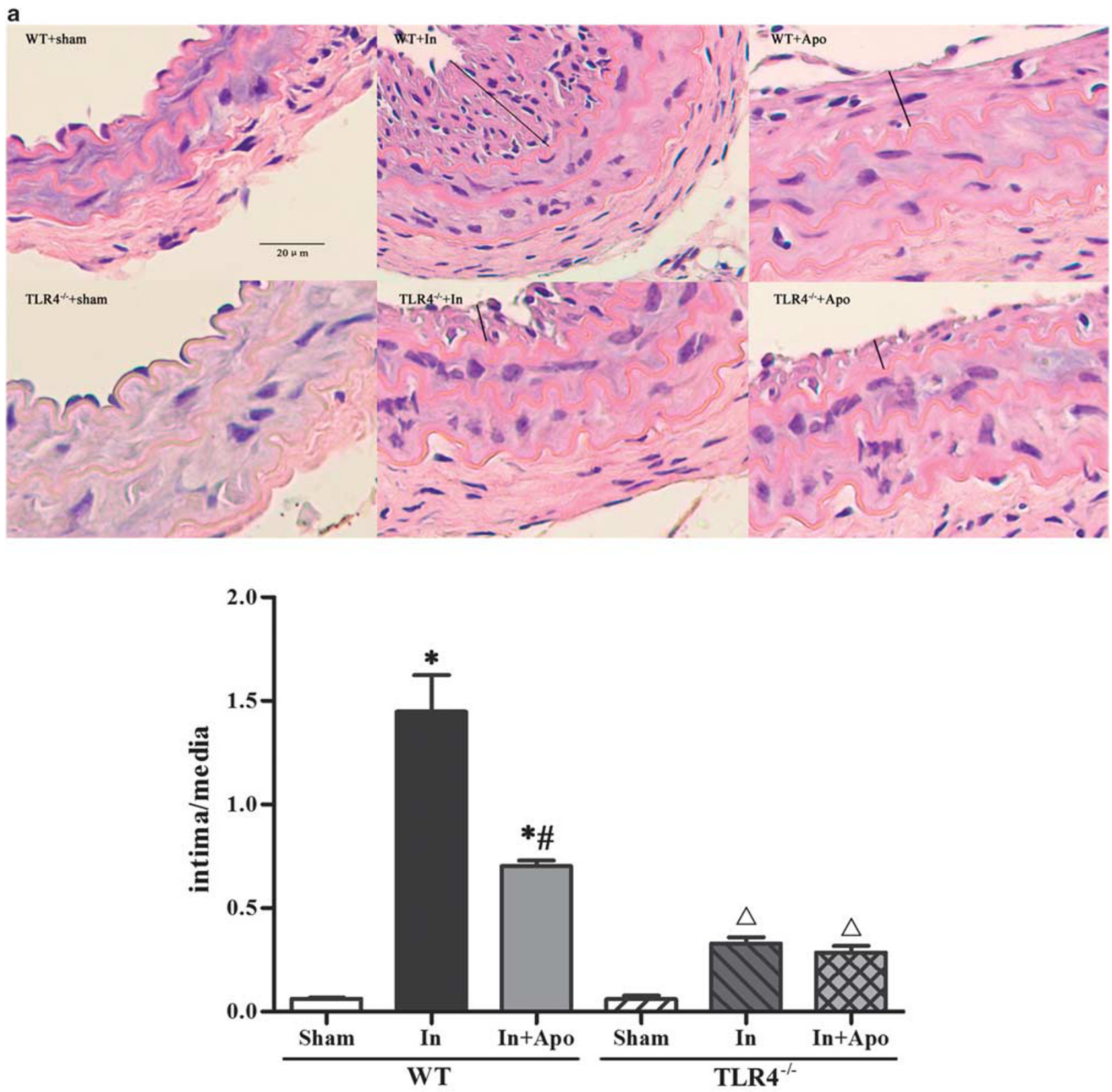

b

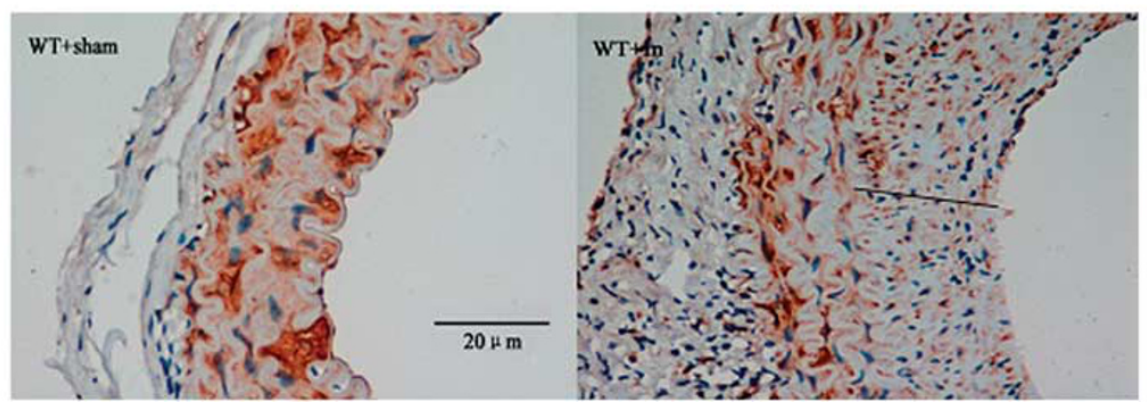

Figure 1 Intimal hyperplasia in carotid arteries. (a) Hematoxylin-eosin staining on cross-sections from representative injured carotid arteries are presented. Carotid wire injury-induced intimal hyperplasia with increased intima/media ratio in WT mice. TLR4 ${ }^{-1-}$ mice displayed a lower intima/media ratio in response to carotid injury. Apo inhibited the wire injury-induced intimal hyperplasia in WT mice but not in TLR4 ${ }^{-1-}$ mice. $(n=3-4$ mice per group. ${ }^{*} P<0.05$ vs WT + sham; ${ }^{\#} P<0.05$ vs WT + In and ${ }^{\triangle} P<0.05$ vs TLR4 ${ }^{-1-}+$ sham.) (b) Accumulation of VSMCs in the neointima identified by $\alpha$-SMA immunostaining. Apo, apocynin; In, carotid wire injury; sham, sham operation; TLR4 ${ }^{-1-}$, TLR4-deficient; WT, wild-type. 


\section{Inhibition of ROS Generation Attenuates TLR4-Mediated Inflammation}

To determine whether carotid injury was associated with systemic inflammation, we measured serum levels of proinflammatory cytokines (IL-1 $\beta$, IL- 6 and TNF- $\alpha$ ) in wire injured and sham-operated mice. As shown in Figure 4a, cytokines expression was elevated at day 14 in sera from injured mice compared with sham-operated mice. Injuryinduced cytokines expression was much higher in WT mice compared with that in TLR4 ${ }^{-1-}$ mice. In addition, the elevated expression of proinflammatory cytokines in WT

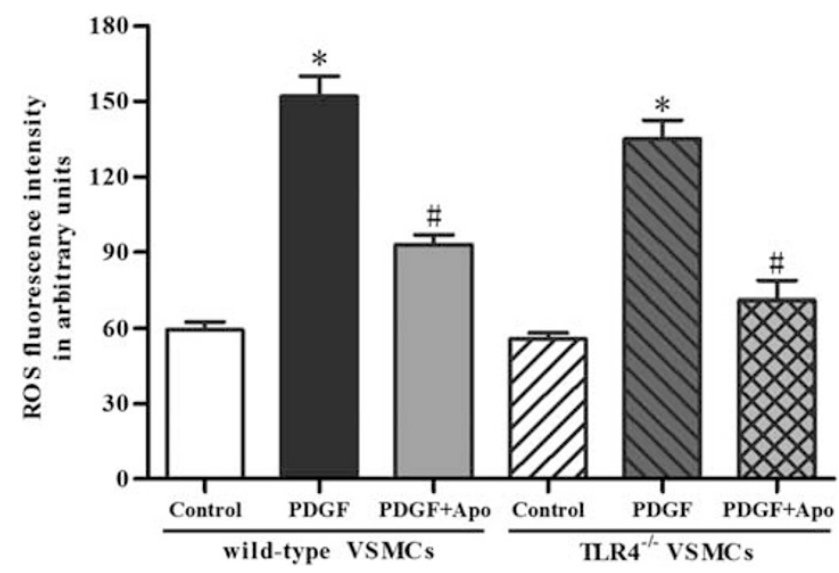

Figure 2 Intracellular ROS generation in VSMCs. VSMCs were treated with PDGF-BB $(20 \mathrm{ng} / \mathrm{ml})$ in the presence or absence of Apo $(100 \mu \mathrm{mol} / \mathrm{l})$ for 60 min. Treatment with PDGF-BB had a significant effect on the basal intracellular ROS generation both in WT and TLR4 ${ }^{-1-}$ VSMCs, and pretreatment with Apo led to suppression of PDFG-BB-induced ROS generation. The intracellular ROS generation was measured by flow cytometry analysis. ( $n=3-4$ cell lines. ${ }^{*} P<0.05$ vs control and ${ }^{\#} P<0.05$ vs PDGF). PDGF-BB, platelet-derived growth factor-BB; ROS, reactive oxygen species; TLR4 ${ }^{-1-}$, TLR4-deficient; VSMC, vascular smooth muscle cell; WT, wild-type. mice was significantly abrogated by apocynin, whereas apocynin did not show any effect on that in TLR4 ${ }^{-1-}$ mice.

Cultured VSMCs in basal conditions displayed very low levels of proinflammatory cytokines. PDGF-BB increased the expression of cytokines especially in cultured WT VSMCs, which were largely retarded by apocynin (Figure $4 \mathrm{~b}$ ). These results appear most consistent with the interpretation that at least part of the suppression of atherogenesis observed in mice/VSMCs with TLR4 deficiency may be mediated by a general reduction in circulating and supernatant levels of proatherogenic inflammatory molecules.

\section{Inhibition of Intracellular ROS Generation Attenuates TLR4-Mediated VSMC Proliferation and Migration}

ROS generation in WT and TLR4 ${ }^{-1-}$ VSMCs was pharmacologically manipulated respectively by PDGF-BB and apocynin, and VSMC proliferation and migration were thereafter detected. Cell proliferation was determined by MTT assay (Figure 5a) and BrdU assay (Figure 5b). Cell migration was measured by modified Boyden chambers (Figure 5c). As shown in Figures $5 a-c$, both WT and TLR4 ${ }^{-1-}$ VSMCs cultured in basal conditions displayed low levels of proliferation and migration. VSMCs from WT mice showed a significant increase in proliferation and migration in response to PDGF$\mathrm{BB}$, whereas VSMCs from TLR4 ${ }^{-1-}$ mice failed to show this increase. However, treatment with apocynin significantly attenuated PDGF-BB-stimulated VSMC proliferation and migration, and this effect was more striking than TLR4 ${ }^{-1-}$ VSMCs. These data suggested that ROS exerts an essential role in the TLR4-mediated VSMC proliferation and migration.

\section{DISCUSSION}

In this study, we have explored the association between ROS generation and TLR4-mediated proinflammatory and proliferative phenotype of VSMCs. VSMCs exposed to PDGF-BB showed significant increase in ROS generation,
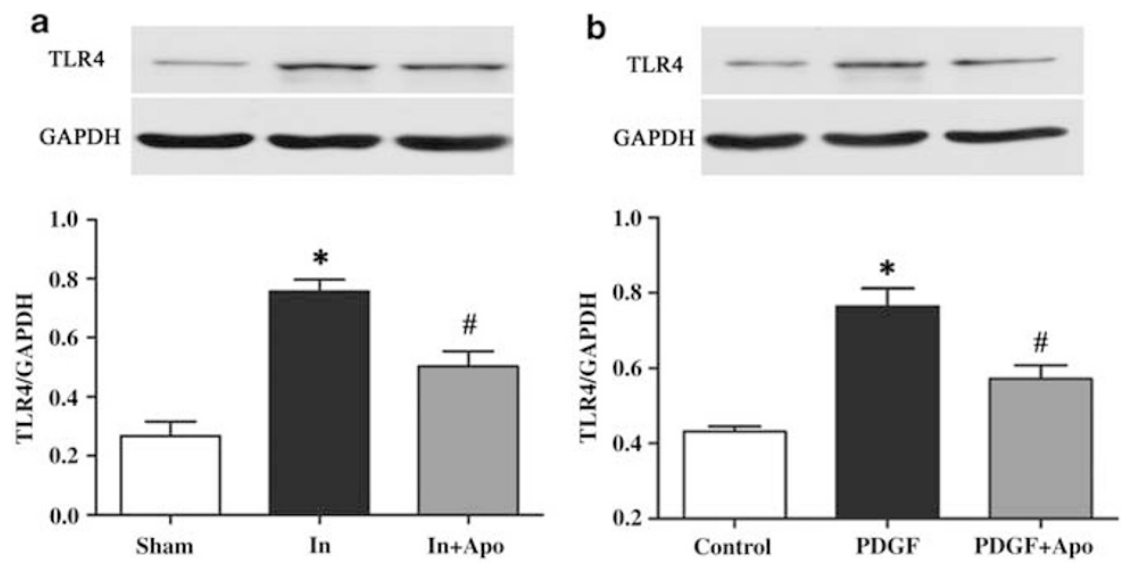

Figure 3 TLR4 expression is dependent on ROS generation. Expressions of TLR4 protein was detected by western blot. (a) Carotid wire injury increased TLR4 protein expression in WT mice and Apo pre-treatment blocked the expression of TLR4 protein. ( $n=3-4$ mice per group. ${ }^{*} P<0.05$ vs sham and ${ }^{\#} P<0.05$ vs In). (b) ROS overproduction with PDGF-BB upregulated TLR4 protein expression whereas ROS inhibition with Apo significantly abrogated this increase in WT VSMCs. ( $n=3-4$ cell lines. ${ }^{*} P<0.05$ vs control and ${ }^{\#} P<0.05$ vs PDGF). In, carotid wire injury; PDGF, platelet-derived growth factor; ROS, reactive oxygen species; sham, sham operation; TLR4, Toll-like receptor 4; VSMC, vascular smooth muscle cell; WT, wild-type. 

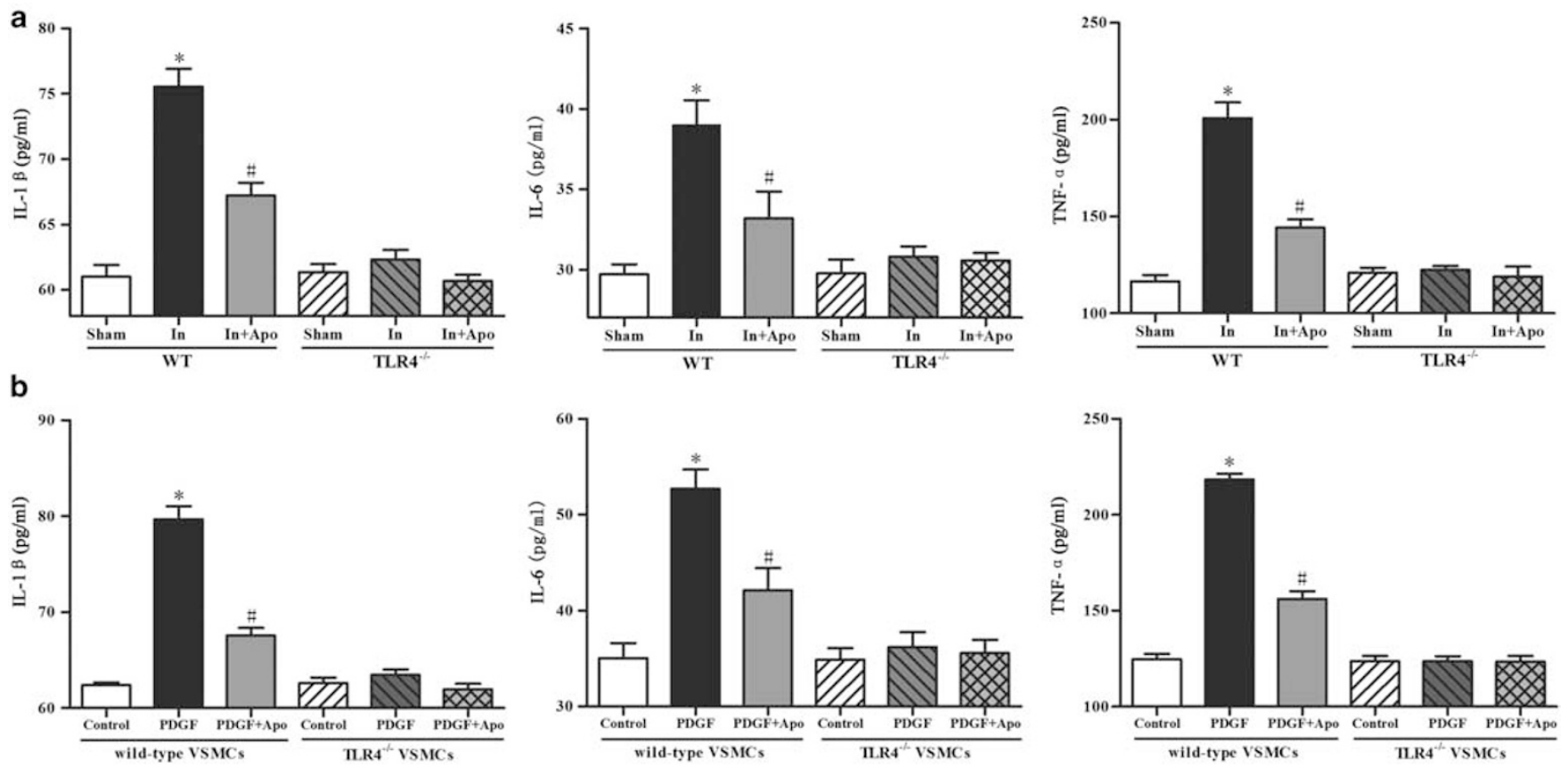

Figure 4 Inhibition of ROS attenuates TLR4-mediated inflammation. Expressions of proinflammatory cytokines (IL-1 $\beta$, IL- 6 and TNF- $\alpha$ ) were detected by ELISA. (a) Carotid wire injury elevated the serum levels of cytokines in WT mice but not in TLR4 ${ }^{-1-}$ mice. Apo pre-treatment blocked the expression of cytokines induced by wire injury in WT mice but not in TLR4 ${ }^{-1-}$ mice. ( $n=3-4$ mice per group. ${ }^{*} P<0.05$ vs WT + sham and ${ }^{\#} P<0.05$ vs WT + In). (b) PDGF-BB upregulated cytokines whereas Apo significantly abrogated this increase in WT VSMCs, but both of them exerted no obvious effect on that in TLR4 ${ }^{-1-}$ VSMCs. $\left(n=3-4\right.$ cell lines. ${ }^{*} P<0.05$ vs WT control and ${ }^{\#} P<0.05$ vs WT PDGF). In, carotid wire injury; IL, interleukin; PDGF-BB, platelet-derived growth factor-BB; ROS, reactive oxygen species; sham, sham operation; TNF- $\alpha$, tumor necrosis factor $\alpha$; TLR4 ${ }^{-1-}$, TLR4-deficient; VSMC, vascular smooth muscle cell; WT, wild-type.

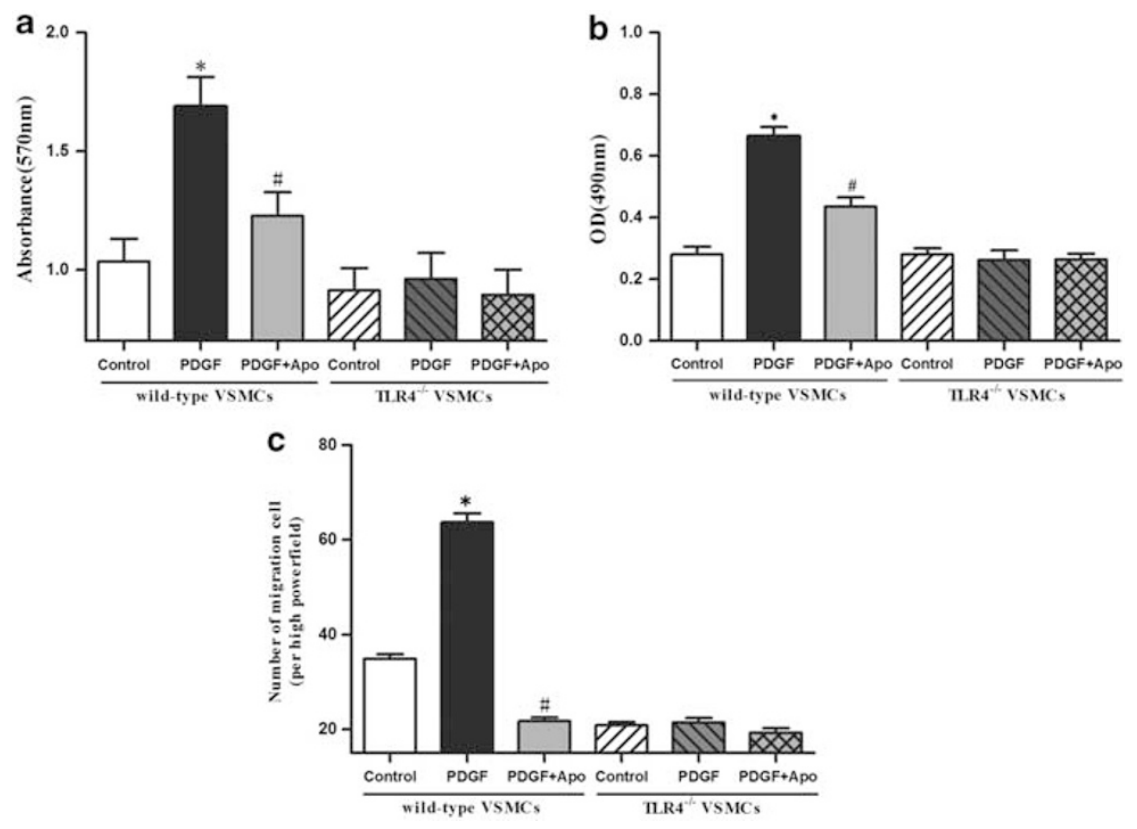

Figure 5 Impact of ROS on VSMC proliferation and migration. Both cultured WT and TLR4 ${ }^{-1}$ VSMCs in basal conditions displayed low levels of proliferation $(\mathbf{a}, \mathbf{b})$ and migration (c), ROS overproduction with PDGF-BB accelerated whereas ROS inhibition with Apo attenuated the VSMC proliferation (a, b) and migration (c). (a) MTT assay; (b) BrdU assay; (c) modified Boyden chambers. ( $n=3-4$ cell lines. ${ }^{*} P<0.05$ vs WT control and ${ }^{\#} P<0.05$ vs WT PDGF). MTT, methyl thiazolyl diphenyl-tetrazolium; OD, optical density; PDGF-BB, platelet-derived growth factor-BB; ROS, reactive oxygen species; TLR4 ${ }^{-1-}$, TLR4-deficient; VSMC, vascular smooth muscle cell; WT, wild-type. 
and this could be effectively suppressed by apocynin. Inhibition of ROS attenuated both TLR4 and proinflammatory cytokines expression in carotid injured mice and VSMCs, and accompanied by a significant inhibition of VSMC proliferation and migration, and neointimal formation. Our studies have provided evidence that ROS may be a key component in TLR4-mediated vascular inflammation and intimal hyperplasia. Inhibiting the primary source of ROS could attenuate TLR4-mediated proinflammatory and proliferative phenotype of VSMCs, and reduce detrimental arterial remodeling and inflammatory reaction such as that in the early stages of atherosclerosis.

Intimal hyperplasia is the common pathological lesion of proliferative vascular diseases, including atherosclerosis, hypertension and restenosis after vascular reconstructive procedures. ${ }^{28}$ Intimal hyperplasia is initiated by endothelial injury or loss, inflammation and stretch. Thereafter, increased VSMC proliferation occurs within 1-3 days, followed by migration of VSMCs into the intima, which eventually contributes to neointimal formation. ${ }^{29}$

An increase in ROS generation is an essential step for development of intimal hyperplasia, atherosclerosis and other vascular pathologies. ${ }^{27}$ ROS accumulation has been demonstrated to have an important role in neointima formation through inducing the proliferation and migration of VSMCs. ${ }^{7-9}$ ROS derived from VSMCs are involved in growth signaling pathways and have an important role in the pathogenesis of several vascular diseases associated with the proliferation and migration of VSMCs. West et $a l^{11}$ and Yokoyama et al ${ }^{12}$ found that intimal VSMCs in hyperplastic and atherosclerotic lesions express high levels of ROS. Consistently, our study found that PDGF-BB, a established stimulant that promotes ROS generation and VSMC viability, ${ }^{10}$ significantly increased ROS generation in VSMCs.

Therefore, inhibition of ROS-stimulated VSMC proliferation, migration and intimal hyperplasia appears to be an important strategy for the prevention of vascular diseases. ${ }^{30}$ In support of this notion, our study showed that pretreatment of VSMCs with apocynin strikingly suppressed PDGF-BB-induced ROS generation. Furthermore, in vivo study showed that apocynin protected wire-injured carotid arteries from intimal hyperplasia. Intensive research efforts have been dedicated to potential mechanisms responsible for the effect of ROS in intimal hyperplasia. It has reported that ROS could induce the VSMC proliferation and migration by enhancing the inflammatory response of ERK1/2 and NF-kappa B pathway, and increasing the expression of mitochondrial biogenesis-related genes. ${ }^{31,32}$ These data further demonstrate the critical role of ROS in VSMC proliferation and migration, and in the neointima formation induced by mechanic injury.

It is widely accepted that inflammation is a major force in the pathophysiology of intimal hyperplasia after vascular injury. TLR4 and its associated proinflammatory cytokines have been shown to promote VMSC proliferation and migration and be closely associated with intimal hyperplasia. Recently, particular attention has been given to the link between TLR4-mediated inflammation and ROS. Inflammatory cytokines stimulate and prime the oxidase and thus contribute to systemic oxidative stress. ROS are reported to be essential in innate immunity, ${ }^{33}$ and related to inflammation, intimal hyperplasia, restenosis, arteriosclerosis and vascular remodeling. ${ }^{34,35}$ ROS were considered to be second messengers in the inflammatory response. ${ }^{36}$ ROS scavenger can reduce ROS generation and attenuate the abnormal production proinflammatory cytokines. ${ }^{13}$ In this study, ROS overproduction increased VSMC proliferation and migration, promoted intimal hyperplasia, and elevated TLR4 and proinflammatory cytokines (IL-1 $\beta$, IL- 6 and TNF- $\alpha$ ) expression in WT mice/ VSMCs, which were attenuated significantly by apocynin. In contrast, TLR4 ${ }^{-1-}$ inhibited carotid wire injury-induced intimal hyperplasia, VSMC proliferation and migration, and proinflammatory cytokines expression. All the previous results suggested that ROS have a key role in the TLR4mediated proinflammatory and proliferative phenotype of VSMCs, and inhibition of ROS could attenuate VSMC proliferation and migration, inflammation, and intimal hyperplasia via a TLR4-mediated signaling pathway.

There is increasing evidence showing that TLR4 can transduce proinflammatory signals produced by ROS, and ROS appear to modulate inflammatory responses through the TLR4-dependent mechanisms. ${ }^{37}$ However, the precise mechanisms by which ROS induced VSMC proinflammatory and proliferative phenotype through TLR4 are not well understood currently. Although ROS may directly affect TLR4 dimerization, an essential step required for initiation of intracellular signaling pathways, it is also possible that ROS may affect TLR4-related signaling pathway through indirect mechanisms. Powers et $a^{38}$ have shown that ROS could increase membrane lipid raft localization of TLR4 during hemorrhagic shock. Of note, Cavassani et al ${ }^{39}$ demonstrated that TLR3 may also be implicated in ROS-dependent inflammation as shown in hyperoxia or intestinal ischemia. Nevertheless, the actual mechanism through which ROS may affect TLR4 signaling pathway, including the extracellular domain of TLR4, remains an important question for future investigation. In this study, we have shown that ROS could regulate the expression of TLR4, and thus affect downstream signaling pathways including the proinflammatory and proliferative phenotype of VSMCs.

\section{CONCLUSIONS}

In conclusion, our study provides the first evidence that inhibition of ROS could attenuate VSMC proliferation, migration and inflammation by suppressing TLR4 expression, and thus attenuate neointima or vascular remodeling following carotid injury. These findings advance our understanding of the pathophysiology function of ROS in vascular disease, and suggest ROS as a perspective target for the prevention and treatment of vascular diseases. 


\section{ACKNOWLEDGEMENTS}

This study was supported by National Natural Science Foundation of China (No. 30970998, No. 81271282), Chongqing Key Project of Science and

Technologies (No. CSTC2010GGC502) and Chongqing Outstanding Youth Science Foundation (No. CSTC2012JJJQ10003).

\section{DISCLOSURE/CONFLICT OF INTEREST}

The authors declare no conflict of interest.

1. Yu H, Payne TJ, Mohanty DK. Effects of slow, sustained, and ratetunable nitric oxide donors on human aortic smooth muscle cells proliferation. Chem Biol Drug Des 2011;78:527-534.

2. Newby AC, Zaltsman AB. Molecular mechanisms in intimal hyperplasia. J Pathol 2000;190:300-309.

3. Antoniades C, Antonopoulos AS, Bendall JK, et al. Targeting redox signaling in the vascular wall: from basic science to clinical practice. Curr Pharm Des 2009;15:329-342.

4. Heistad DD, Wakisaka Y, Miller J, et al. Novel aspects of oxidative stress in cardiovascular diseases. Circ J 2009;73:201-207.

5. Lang $Y, C$ Chen $D$, Li D, et al. Luteolin inhibited hydrogen peroxideinduced vascular smooth muscle cells proliferation and migration by suppressing the src and akt signalling pathways. J Pharm Pharmaco 2012;64:597-603.

6. Song $\mathrm{P}$, Zou $\mathrm{MH}$. Regulation of $\mathrm{Nad}(\mathrm{P}) \mathrm{H}$ oxidases by ampk in cardiovascular systems. Free Radic Biol Med 2012;52:1607-1619.

7. Panchenko MP, Silva N, Stone JR. Up-regulation of a hydrogen peroxide-responsive pre-MRNA binding protein in atherosclerosis and intimal hyperplasia. Cardiovasc Pathol 2009;18:167-172.

8. Wu L, Li X, Li Y, et al. Proliferative inhibition of danxiongfang and its active ingredients on rat vascular smooth muscle cell and protective effect on the vsmc damage induced by hydrogen peroxide. J Ethnopharmacol 2009;126:197-206.

9. Touyz RM, Briones AM. Reactive oxygen species and vascular biology: implications in human hypertension. Hypertens Res 2011;34:5-14.

10. Qin ZX, Yu P, Qian DH, et al. Hydrogen-rich saline prevents neointima formation after carotid balloon injury by suppressing ROS and the TNF-alpha/NF-kappab pathway. Atherosclerosis 2012;220:343-350.

11. West $N$, Guzik T, Black $E$, et al. Enhanced superoxide production in experimental venous bypass graft intimal hyperplasia: role of $\mathrm{NAD}(\mathrm{P}) \mathrm{H}$ oxidase. Arterioscler Thromb Vasc Biol 2001:21:189-194.

12. Yokoyama $M$, Inoue $N$, Kawashima $S$. Role of the vascular NADH/ $\mathrm{NADPH}$ oxidase system in atherosclerosis. Ann $\mathrm{N}$ Y Acad Sci 2000;902:241-247, discussion 247-248.

13. Kim JY, Park HJ, Um SH, et al. Sulforaphane suppresses vascular adhesion molecule-1 expression in TNF-alpha-stimulated mouse vascular smooth muscle cells: involvement of the MAPK, NF-kappab and AP-1 signaling pathways. Vascul Pharmacol 2012;56:131-141.

14. Sadowitz B, Seymour K, Gahtan V, et al. The role of hyaluronic acid in atherosclerosis and intimal hyperplasia. J Surg Res 2012;173:e63-e72.

15. Sheehan AL, Carrell S, Johnson B, et al. Role for NOX1 NADPH oxidase in atherosclerosis. Atherosclerosis 2011;216:321-326.

16. Dasu MR, Devaraj $S$, Zhao $L$, et al. High glucose induces TOLL-like receptor expression in human monocytes: mechanism of activation. Diabetes 2008;57:3090-3098.

17. Zhang $L L, G a o C Y$, Fang $C Q$, et al. Ppargamma attenuates intimal hyperplasia by inhibiting TLR4-mediated inflammation in vascular smooth muscle cells. Cardiovasc Res 2011:92:484-493.

18. Yang X, Coriolan D, Murthy V, et al. Proinflammatory phenotype of vascular smooth muscle cells: role of efficient Toll-like receptor 4 signaling. Am J Physiol Heart Circ Physiol 2005;289:H1069-H1076.

19. de Graaf R, Kloppenburg G, Kitslaar PJ, et al. Human heat shock protein 60 stimulates vascular smooth muscle cell proliferation through toll-like receptors 2 and 4. Microbes Infect 2006;8:1859-1865.
20. Fan J, Frey RS, Malik AB. TLR4 signaling induces TLR2 expression in endothelial cells via neutrophil nadph oxidase. J Clin Invest 2003; 112:1234-1243.

21. Faure $\mathrm{E}$, Thomas $\mathrm{L}, \mathrm{Xu} \mathrm{H}$, et al. Bacterial lipopolysaccharide and IFN-gamma induce Toll-like receptor 2 and Toll-like receptor 4 expression in human endothelial cells: role of NF-kappa b activation. J Immunol 2001;166:2018-2024.

22. Lindner V, Fingerle J, Reidy MA. Mouse model of arterial injury. Circ Res 1993;73:792-796.

23. Heumuller S, Wind S, Barbosa-Sicard E, et al. Apocynin is not an inhibitor of vascular nadph oxidases but an antioxidant. Hypertension 2008;51:211-217.

24. Patterson C, Ruef J, Madamanchi NR, et al. Stimulation of a vascular smooth muscle cell $\mathrm{NAD}(\mathrm{P}) \mathrm{H}$ oxidase by thrombin. Evidence that p47(phox) may participate in forming this oxidase in vitro and in vivo. J Biol Chem 1999;274:19814-19822.

25. Ellmark SH, Dusting GJ, Fui MN, et al. The contribution of NOX4 to NADPH oxidase activity in mouse vascular smooth muscle. Cardiovasc Res 2005;65:495-504.

26. Lin FY, Chen YH, Tasi JS, et al. Endotoxin induces Toll-like receptor 4 expression in vascular smooth muscle cells via NADPH oxidase activation and mitogen-activated protein kinase signaling pathways. Arterioscler Thromb Vasc Biol 2006;26:2630-2637.

27. Martinez-Outschoorn UE, Lin Z, Trimmer C, et al. Cancer cells metabolically 'Fertilize' The tumor microenvironment with hydrogen peroxide, driving the warburg effect: implications for pet imaging of human tumors. Cell Cycle 2011;10:2504-2520.

28. Zhang LN, Parkinson JF, Haskell C, et al. Mechanisms of intimal hyperplasia learned from a murine carotid artery ligation model. Curr Vasc Pharmacol 2008;6:37-43.

29. Hlawaty $H$, Suffee $N$, Sutton $A$, et al. Low molecular weight fucoidan prevents intimal hyperplasia in rat injured thoracic aorta through the modulation of matrix metalloproteinase-2 expression. Biochem Pharmacol 2011;81:233-24.

30. Limon-Pacheco J, Gonsebatt ME. The role of antioxidants and antioxidant-related enzymes in protective responses to environmentally induced oxidative stress. Mutat Res 2009;674:137-147.

31. Zhang J, Chen J, Yang J, et al. Resveratrol attenuates oxidative stress induced by balloon injury in the rat carotid artery through actions on the ERK1/2 and NF-kappa b pathway. Cell Physiol Biochem 2013; 31:230-241.

32. Abhijit S, Bhaskaran R, Narayanasamy A, et al. Hyperinsulinemiainduced vascular smooth muscle cell (VSMC) migration and proliferation is mediated by converging mechanisms of mitochondrial dysfunction and oxidative stress. Mol Cell Biochem 2013;373:95-105.

33. Streeter J, Thiel W, Brieger K et al. Opportunity NOX: the future of nadph oxidases as therapeutic targets in cardiovascular disease. Cardiovasc Ther 2012;31:125-137.

34. Brandes RP, Weissmann N, Schroder K. NADPH oxidases in cardiovascular disease. Free Radic Biol Med 2010;49:687-706.

35. Sirker A, Zhang M, Shah AM. NADPH oxidases in cardiovascular disease: insights from in vivo models and clinical studies. Basic Res Cardiol 2011;106:735-747.

36. Vega A, Chacon P, Alba G, et al. Modulation of IgE-dependent cox-2 gene expression by reactive oxygen species in human neutrophils. J Leukoc Biol 2006;80:152-163.

37. Lorne $\mathrm{E}$, Dupont $\mathrm{H}$, Abraham E. Toll-like receptors 2 and 4: initiators of non-septic inflammation in critical care medicine? Intensive Care Med 2010;36:1826-1835

38. Powers KA, Szaszi K, Khadaroo RG, et al. Oxidative stress generated by hemorrhagic shock recruits Toll-like receptor 4 to the plasma membrane in macrophages. J Exp Med 2006;203:1951-1961.

39. Cavassani KA, Ishii $M$, Wen $H$, et al. TLR3 is an endogenous sensor of tissue necrosis during acute inflammatory events. J Exp Med 2008;205:2609-2621. 